\title{
Automatic Verification of Communicative Commitments using Reduction
}

\author{
Mofleh Al-diabat \\ Department of Computer Science, Al Albayt University, Jordan \\ moflehd@aabu.edu.jo \\ Faisal Al-saqqar \\ Faculty of Engineering, Concordia University, United States \\ falsaqq@encs.concordia.ca \\ Ashraf Al-saqqar \\ Irbid Electricity Company, Irbid-Jordan \\ ashraf.saqqar@ideco.com.jo
}

\begin{abstract}
Albeit the modeling and verification of the Multi-Agent Systems (MASs) have been since long under study, there are several related challenges that should still be addressed. In effect, several frameworks have been established for modeling and verifying the MASs with regard to communicative commitments. A bulky volume of research has been conducted for defining semantics of these systems. Though, formal verification of these systems is still unresolved research problem. Within this context, this paper presents the CTLcom that reforms the CTLC, i.e., the temporal logic of the commitments, so as to enable reasoning about the communication and fulfillment of commitments. Moreover, the paper introduces a fully-automated method for verification of the logic by means of trimming down the problem of a model that checks the CTL com to a problem of a model that checks the GCTL", which is a generalized version of the CTL" with action formulae. By so doing, we take advantage of the CWB-NC automata-based model checker as a tool for verification. Lastly, this paper presents a case study drawn from the business field, that is, the NetBill protocol, illustrates its implementation, and discusses the associated experimental results in order to illustrate the efficiency and effectiveness of the suggested technique.
\end{abstract}

Keywords: Multi-Agent Systems, Model Checking, Communicative Commitments, Reduction

Received: 13 April 2018, Revised 22 May 2018, Accepted 1 June 2018

\section{DOI:}

(C) 2018 DLINE. All Rights Reserved

\section{Introduction}

Communication in the Multi-Agent Systems (MASs) is a key process by which the agents coordinate their actions and behaviors to achieve their goals [50]. However, in order for autonomous and heterogeneous agents to interact with each other, they use Agent Communication Languages (ACLs), which are usually associated with a semantic memory composed of different ontologies [21, 19]. Making this communication possible raises a need for defining formal semantics for these ACLs [25]. The first attempt to define such semantics was the Speech Acts Theory of Searle [38]. The formal semantics of Searle were described as the mental approach that attempts to make rational balance between certain agent communication notions like intention, belief, and desire. The mental approach suggests that the agents can read the minds of one the other [41]. Hence, the mental approaches cannot verify whether or not an agent is functioning in accordance with given semantics, which is a problem known commonly as the ACL semantics verification problem [49].

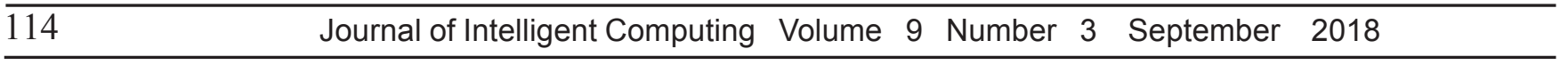


Thus, a switch in the MAS community to the social approaches took place so as to outdo the drawbacks of the semantics of the ACLs that are defined by using mental approaches [41]. The social approaches, on the other hand, are employed for defining formal semantics for the ACLs $[1,23,30,43,52]$. Social commitments are employed in a number of those social approaches that successfully render robust representations to model the multi-agent interactions $[8,17,18,20,33,43]$. This paper uses communicative social commitments as defined in $[6,25,27]$ as a tool for information transfer via message passing. These commitments are formally referred to by $C_{i} \rightarrow j \phi$, which means that the agent $i$, which makes the commitment (i.e., the debtor), commits to the agent $j$, namely, the creditor (i.e., the agent for which the commitment is made), that content of commitment ( $\phi)$ holds [27].

Example 1. A merchant, $\mathrm{M}$ er, commits to deliver goods to customer, Cus. This commitment is expressed as $\mathrm{CM}$ er $\rightarrow \mathrm{Cus} \phi$, where $\phi$ means 'deliver the due goods'.

During the past decade, the social commitments were efficiently used in a broad range of fields that range from development of artificial institutions [31], to modeling business processes [18], development of Web-based applications [45], and specification and modeling of multi-agent interaction protocols, known as commitment-based protocols $[4,17,37,52]$. The commitment between agents is not simply a static entity. Rather, it is a dynamic entity whose state changes with time [32]. This dynamicity characteristic supports flexibility of the commitments. In addition, it, can be captured by manipulating the commitments by means of certain operations like discharge, creation, release, cancellation, delegation, and assignment [42].

This ability to manipulate and negotiate the commitments is actually the most prominent element that makes the commitmentbased approaches quite flexible and powerful in capturing various interaction patterns. On the other hand, the commitment-based semantics for the ACL messages should fulfill the following critical criteria [43]:

Being formal, that is, the semantics should be based on a formal language, for instance, temporal logic;

Being declarative, i.e., the semantics should focus on the meanings of the messages rather than on how the messages are exchanged;

Being verifiable, that is, the semantics should enable the check of whether the agents are functioning in accordance with the semantics or not);

Being meaningful, namely, the semantics should concentrate on the message content rather than on representation of them as tokens).

However, the incentive is not only inference about the social commitments and their actions, but is also application of the automatic and formal verification methods, like model checking, so as to verify the commitments. In the open environments like the e-business context, it is actually not realistic to presume that all the autonomous agents will act in accordance with the given protocols because it is likely that they will not function according to their commitments. Furthermore, formal verification is necessary for helping the protocol designers to enforce desired agent behaviors so that these protocols adhere to the given specifications at the design time.

Various approaches have successfully addressed the aforementioned challenge, including (i) the local testing method [45], (ii) the static verification method [37, 52], and (iii) the semi-automatic verification method [51], in order to identify the non-compliant and compliant agents at the ends of these protocols.

In other respects, modeling the MASs when there are numerous dimensions to consider simultaneously is challenge that makes their verification a hard task [5,34].

This paper aimed at (i) introducing a CTLcom logic by redefining the social accessibility relationship given in [6, 25] so as to account for the intuition that the social commitments are transferred through communication between agents, (ii) refining the semantics of the commitments and their fulfillment that were first defined in [6], (iii) developing automated mechanism for trimming down the problem from the problem of a model that checks the CTLcom to the problem of model that checks the GCTL*, (iv) proving soundness of the suggested reduction method, and (v) applying the proposed reduction procedure on real case study; the NetBill (NB) protocol [44], from the e-business field, and discussing the verification results. 
2. The rest of this paper is organized as follows. Section 2 highlights and discusses related previous works. Section 3 introduces the interpreted system formalism, its extended communicative version, and our model. Then, the semantics and syntax of CTLcom are discussed in Section 4 while the model checking problem of CTL com is illustrated in Section 5. Afterwards, Section 6 is devoted to a discussion of implementation of the proposed model where as Section 7 concludes this paper.

\section{Related Previous Works}

After introducing to integration of the social commitment concept by Singh in $[39,40]$, research on the agent communication language on the basis of the commitment had gone along way. Singh was the first to figure out the importance of incorporating the social notion of commitments to the agents in ACLs. He differentiated between two kinds of commitments: psychological commitment (i.e., a commitment of an agent to herself/himself) as it is exploited in the Artificial Intelligence (AI), and social commitment (i.e., a commitment of one agent to another to do certain actions). Singh concluded that the psychological commitment is a very restricted form of commitments as it corresponds to a unidirectional relationship and, once committed to a certain be lie for intention, the agent cannot reconsider it, even if she/he gets some positive new evidence or if the commitment contradicts with her/his goal. Later, Singh's social notion of commitment was further investigated by Castel franchi [14], with a focus on the interactions among members of groups and organizations from a social perspective. Castel franchi paid attention to clarification of some concepts to be able to suggest descriptive ontology to the organizations theory without taking into account the computation al facets of social commitments. However, the first attempt to define these mantics of ACLs in terms of social concepts was made by Singh in[41]. This attempt emphasized the conventional meaning and public perspective. Since then, social commitments in have been mostly defined for agent communication in terms of computational logics [9, 11, 43, 46]. However, other researchers have proposed different techniques such as event calculus [52]. In this section, we are primarily interested in those approaches in which commitments are defined in terms of computational logics.

In [43], Singh extended the CTL logic with modalities for social intentions, beliefs, and commitments so as to formally model the interactions between the interacting agents in the MAS. By so doing, he could develop a specification language for the commitment-based protocols. He defined three accessibility relationships so as to intuitively capture the meanings of the new modalities. Regarding the commitment, he claimed that a commitment is fulfilled at specific state if, and only if (iff), its content is true along all the accessible paths which have been defined by an accessible relationship emanates from the commitment state, i.e., the state in which the commitment holds.

In[46], Verdicchio and Colombetti proposed a logical frame work for definition of the ACL semantics on the basis of the social commitment concept. In addition, they suggested formal definitions of these actions with in the context fatemporal logic that expands the $\mathrm{CTL}^{*}$ with past-oriented temporal operators. In this suggested system, which is called $\mathrm{CTL}^{ \pm}$, time is supposed to be discrete, having no starting or end point, and branching in the future.

The $\mathrm{CTL}^{ \pm}$is then stretched to represent commitments and actions.

Bentahar el al. $[10,11]$ introduced a method that extends the $\mathrm{CTL}^{*}$ with an operator for the commitments and their actions and two operators for the argument and the dynamic logic. For defining semantics for the commitment modality, they formulated a new definition for the accessibility relationships. Furthermore, they defined the semantics in terms of the computations (namely, paths) along which commitment is met, which is more intuitive than the semantics introduced by Singh in [43]. However, the semantics of the associated actions were defined recursively (i.e., semantics of every action are dependent on those of the other actions, which made verification of the suggested logic quite a challenge.

El-Menshawy et al. [24] tried to overcome the limitations raised in $[10,11]$ by suggesting new logical language called CTL"sc to develop specification language for the commitment-based protocols. The proposed logic expands the CTL"with commitments and concomitant actions. Additionally, they expanded the temporal modalities of the CTL ${ }^{*}$ with past-oriented temporal modalities. Semantics of the actions were not defined in recursive way as in $[10,11]$, that is, semantics of every action are independent of those of other actions.

Later, El-Menshawy et al. [28] defined new temporal logic called the CTLC by expanding the CTLwith operators for the social commitments, as well as for their fulfillments and violations. Their main contribution was defining new social accessibility relationship whereby that presumed presence of intermediate state between the commitment and fulfillment states. However,

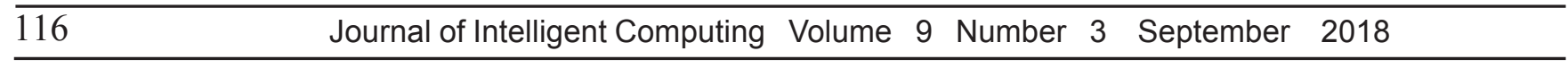


introduction of the intermediate state makes computation of the accessible states very complex.

The CTLC logic has also been the basis of the works presented in Bentahar et al. [6] and El-Menshawy et al. [25]. In [6], Bentahar et al. refined the CTLC by introducing a number of unshared and shared variables so that their expanded version of interpreted systems can explain the communication between the interacting agents. What is particularly appealing in their approach is that the shared variables are only employed for motivating presence of communication channels, not for establishment of a communication. In [25], on the other hand, the researchers modified the CTLC into the $\mathrm{CTLC}^{+}$which enables reasoning about the communicating commitments and their fulfillments. In this paper, we modify the social accessibility relationship presented in [6, 25] by allowing both agents to use their unshared variables in the communications with other agents (i.e., establishing more commitments). Details about our new social accessibility relation are given in Section 3.

In [26], El-Menshawy et al. proposed new logic-based language for specifying the commitment-based protocols. They defined this language in terms of the $\mathrm{ACTL}^{* c}$ logic, which, subsequently, expands the $\mathrm{CTL}^{*}$ with operators for the social commitments and associated actions.

\section{InterpretedSystemsand the CTL com logic}

This section summarizes formalism of the interpreted systems that was introduced in [29] to model the MASs, besides two extensions of this formalism, in order to account for the agent's communication by use of the social commitments given by $[6,25]$. Thereafter, we introduce our new social accessibility relationship.

\subsection{Interpreted Systems}

Formalism of the interpreted systems, which was introduced by Fagin et al. in [29], represents useful framework for locally modeling the heterogeneous and autonomous agents that interoperate within a global system by sending and receiving messages. Such formalism models different classes of MASs like the asynchronous and synchronous systems. We support this formalism for the following three reasons:

1. It is useful for relating autonomous and social behaviors to MAS components [27].

2. It enables abstraction from details of the components of the system and concentrates only on the interactions among the communicating parties.

3. It supports interoperability between the global and local models [29].

Assume having a set $A g=\{1, \ldots, n\}$ of $n$ agents. At all the times, every agent in the system is supposed to be ina certain local state that intuitively records all the information accessible for the agent at that time. In specific, every agent $i \in A g$ is distinguished with countable sets $L i$ and $A c t i$ of local states and actions, respectively, in which Acti is primarily employed to explain the temporal evolution of the system. Furthermore, for every $i \in A g$ the local actions are implemented in agreement with a local protocol $P i: L i$ $\rightarrow 2 A c t i$, which determines a group of enabled local actions in a specified local state. Moreover, the environment wherein the agents live may be modeled by a special agent $e$. A group of local states, $L e$, actions, $A c t e$, and protocol, $P e$, are usually associated with $e$. A tuple $g=(l 1, \ldots, l n, l e) \in(L 1 \times \ldots \times L n \times L e)$ where $l i \in L i$ for each $i \in A g t$ and $l e \in L e$, is called a 'global state' and represents the immediate arrangement of all the agents in the system at a specified time.

The local evolution function $T i$ which specifies the transitions for an agent, $i$, amongst its local states is defined according to the equation:

$$
\tau_{i}: L i \times L e \times A c t i \rightarrow L i
$$

Likewise, the global evolution function of the system is given by:

$$
\tau: G \times A C T \rightarrow G
$$

where $A C T=A c t 1 \times \ldots \times A c t n$ and every component $a \in A C T$ is termed 'joint action'. It is a tuple of actions (one for every agent) and $G=L 1 \times \ldots \times L n \times L e$ expresses a group of global states. The symbol $l i(g)$ is employed to represent the local state of the agent $i$ in the global state $g$. As well, $I \in G$ is a starting global state for this system. For the purpose of simplification, we exclude the 
environment agent from the interpreted formalism of the system as was performed in [35].

Bentahar et al. [6] and El-Menshawy et al. [25] expanded Fagin et al.'s formalism of the interpreted systems with unshared and shared variables so as to acknowledge the communication which takes place during execution of the MASs and to give intuitive semantics for the social commitments which are established by the communications between the interacting agents. In specific, they associated a countable group, $V$ ari, of local variables with every agent $i \in A g t$. They, then, utilized these variables to represent the communication channels through which the messages are sent and received. From the technical point of view, they referred to the value of a variable, $x$, in the set $V$ ari in the local state $l i(g)$ by $l x(g)$. Accordingly,

$$
\text { if } l i(g)=l_{i}(g 1), \text { then } l x(g)=l x(g 1) \text { for all } x \in \text { Vari }
$$

The point is that for any two agents, $i$ and $j$, to communicate, they have to share a communication channel that is represented by shared variables between $i$ and $j$. Our model, $M$, is generated from the formalism introduced by [29] and the extension we have introduced.

Definition 1: (Model of CTLcom). A model set of all models $M$ is a tuple, where:

- $S \subseteq L 1 \times \cdots \times L n$ is the set of the global states that are reachable by the system.

- $I \subseteq S$ is a set of starting global states for the system.

- $R t \subseteq S \times S$ is the transition relationship that is defined by $(s, s 1) \in$ Rtiff joint action exists:

$(a 1, \ldots, a n) \in A C T$ such that $\tau(s, a 1, \ldots, a n)=s 1$.

For each pair $(i, j) \in A g 2, \sim i \rightarrow j \subseteq S \times S$ is the social accessibility relation defined by $s \sim i \rightarrow j s 1$

iff $V$ ari $\cap V \operatorname{arj} /=\varnothing$ such that $\forall x \in V$ ari $\cap V$ arj

we have $l x(s)=l x(s 1)=l x(s 1)$.

$\begin{array}{lll}I & j & i\end{array}$

- $V: S \rightarrow 2 \phi p$ is a valuation function where $\phi p$ is a set of atomic propositions.

In this model, $M$, we assume that each accessible state is reachable. Intuition behind the new social accessibility relationship $\sim i$ $\rightarrow j$ from one global state, $s$, to another $s 1(s \sim i \rightarrow j s 1)$ is that there exist certain shared variables, that is, a communication channel, between $i$ and $j$ such that the agent $i$ fills the channel in $s$, and the agent $j$ receives the content of the channel in $s 1$. After receiving the content of the channel, all variables shared between $i$ and $j$ will have the same values

$$
\begin{aligned}
& \text { (i.e., } l x(s)=l x(s 1)=l x(s 1) \forall x \in \\
& i \quad i \quad j \\
& \text { Vari } \cap \text { Varj ) }
\end{aligned}
$$

Dissimilar with the social accessibility relationship in [6,25], there is no restriction on content of the unshared variables for the two agents since they can receive information at the same time from other agents via other channels that involve other variables for every one of these two agents. Therefore, these variables can differ from $s$ to $s 1$ This concept is explained in Figure 1, where two agents, $i$ and $j$, are communicating through a channel and their unshared and shared are the following:

Agent $i$ :

Vari $=\{x 1, x 2, x 3\} ;$ Agent $j: \operatorname{Varj}=\{x 1, x 1, x 1\}$.

The variable $x 1$ is the variable shared by the two agents. It denotes presence of communication channel between $i$ and $j$.

The variables $x 2, x 3, x$ and $x 1$ are the variables unshared by the two agents. When the communication channel is established, the value of $x 1$ for agent $j$ in $s$ is changed to be equal to the value of variable $x 1$ for agent $I$ in $s 1$. This illustrates the message passing through the channel. 


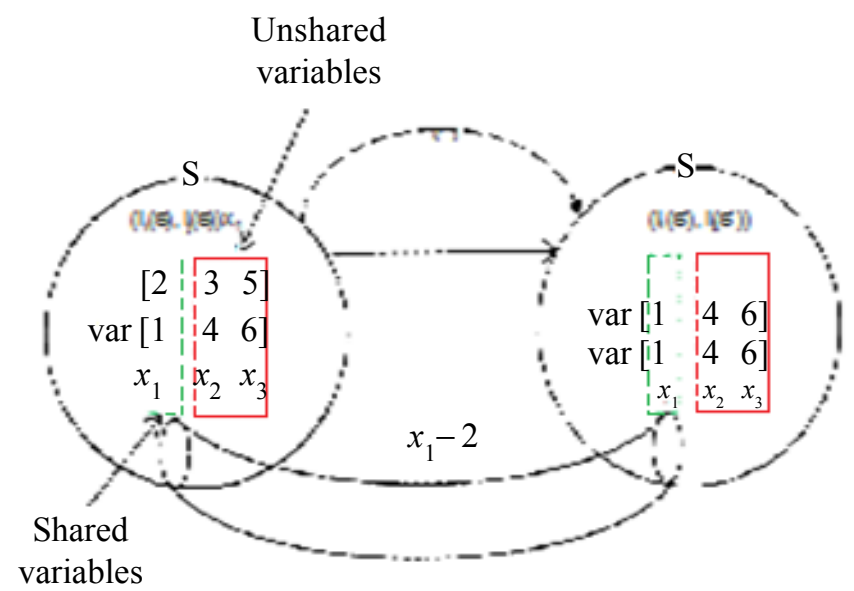

Figure 1. An example of social accessibility relation $\sim i \rightarrow j$

Thus, the main motivation behind defining $\sim i \rightarrow j$ over the social accessibility relation in $[6,25]$ is that the definition of the accessibility relation in $[6,25]$ over specifies and over constrains the concept of communicative commitments in the sense that the state accessible for the debtor $i$ should be the same. Notice that not only can the creditor $\mathrm{j}$ gain more information when moving from one state to an accessible one, but also the debtor i can change its local state by probably gaining new information through other communications. Therefore, only the shared variables should be the same. Moreover, by relaxing this condition of having the same values for unshared variables, we allow the agent to establish and participate in multiple communications while being in the same state.

\section{CTL ${ }^{\text {com }}$ Logic}

This section presents the semantics and syntax of the CTLcom logic, which is a combination of the branching time temporal logic CTL $[2,16]$ with two modalities to figure out the social commitments and their fulfillments. The syntax of CTLcom is defined as follows:

$\varphi::=p|\neg \varphi| \varphi \vee \varphi|E X \varphi| E(\varphi \mathrm{U} \varphi)|E G \varphi| C i \rightarrow j \varphi \mid F u(C i \rightarrow j \varphi)$.

where

- $p \in \phi p$ is atomic proposition;

- The Boolean connectives $\neg$, and $V$ is defined in the usual manner;

- $E$ is the existential quantifier on paths;

- $X, U$, and $G$ are $C T L$ path modal connectives standing for 'next', 'until', and 'globally', respectively;

- The modal connective $C i \rightarrow j$ expresses 'commitment from $i$ to $j$ '; and

- The modal connective $F u$ stands for 'fulfillment'.

According to this logic, $C i \rightarrow j \varphi$ is read as 'the agent $i$ commits to the agent $j$ to result in $\varphi$ '. $F u(C i \rightarrow j \varphi)$ is read as 'commitment $C i \rightarrow j \varphi$ has been met'.

Other temporal modalities, e.g., $F$ (future), and the universal path quantifier $A$ can be defined as usual according to the foregoing definition (e.g., [16]). 
A path $\pi=(s 0, s 1, \ldots)$ in a model $M$ is infinite sequence of global states that are reachable in $S$ such that for all $i>0,(s i, s i+1) \in$ Rt. $\pi(i)$ indicates the $(i+1)^{\text {th }}$ state of $\pi(i . e ., \pi(i)=s i)[3]$.

Definition 3 (Satisfiability and Validity).

A formula $\varphi$ is judged to be satisfied (expressed as $(M, s) \mid=\varphi$ ) when a model $M$ exists and a state $s$ for which $\varphi$ holds. A formula $\varphi$ is valid (written as $\mid=\varphi$ ) iff $(M, s) \mid=\varphi$ for all models $M \in M$ and for all $s \in S$.

Definition 4 (Satisfaction of CTLcom).

Given model $M$, satisfaction of the CTLcom formula $\varphi$ in global state, $\mathrm{s}$, expressed as $(M, s) \mid=\varphi$, is defined recursively as follows:

$\bullet(M, s) \mid=p$ iff $p \in \mathrm{V}(s)$

$\bullet(M, s) \mid=\neg \varphi \operatorname{iff}(M, s)^{*} \varphi$

$\bullet(M, s)|=\varphi \operatorname{V} \psi \operatorname{iff}(M, s)|=\varphi$ or $(M, s) \mid=\psi$;

$\bullet(M, s) \mid=E X \varphi$ iff there is a path $\pi$ beginning at $\mathrm{s}$ such that $(M, \pi(1)) \mid=\varphi$;

$\cdot(M, s) \mid=E(\varphi \mathrm{U} \psi)$ iff there is a path $\pi$ that starts at s such that for $k \geq 0,(M, \pi(k)) \mid=\psi$ and $(M, \pi(j)) \mid=\varphi$ for all $0 \leq j<k$;

$\cdot(M, s) \mid=E G \varphi$ iff there is a path $\in$ beginning at $s$ such that $(M, \pi(k)) \mid=\varphi$ for all $k \geq 0$;

$\cdot(M, s) \mid=C i \rightarrow j \varphi$ iff for all the global states $s 1 \in S$ such that $s \sim i \rightarrow j s 1$, we have $(M, s 1) \mid=\varphi$;

$\cdot(M, s) \mid=F u(C i \rightarrow j \varphi)$ iff there is $s 1 \in S$ such that $s 1 \sim i \rightarrow j s$ and $(M, s 1) \mid=C i \rightarrow j \varphi$.

Semantics of the CTLcom state formula are defined in model $M$ as usual (the semantics of the CTL (e.g., $[2,16])$ ) besides two modalities for the commitments and their fulfillment. In these semantics, the formula of the state, $C i \rightarrow j \varphi$, is fulfilled in model $M$ at $s$ iff the content $\varphi$ holds in each accessible state $s 1$ achieved by the social accessibility relationship $\sim i \rightarrow j$. Intuition of these semantics is the following: when $i$ commits to $j$ using a communication channel, the variables shared by $i$ and $j$ will all have similar values. There is no limitation on content of the unshared variables for the two agents owing to that they can receive information at the same time from other agents via other channels that involve other variables for each of them. Thus, these variables can differ from $s$ to $s 1$. This explains the message that passes through the channel. The state formula $F u(C i \rightarrow j \varphi)$ is fulfilled in the model $M$ iff there is a state $s 1$ that satisfies the commitment from which $s$ can be observed by using the social accessibility relationship $\sim i \rightarrow j$.

\section{Model Checking CTLcom Using Reduction}

This section presents new technique for modeling check CTLcom. In a nutshell, the technical formulation of the CTLcom model checking problem is as follows: Given a MAS that is represented as interpreted system model $M$ and a formula $\varphi$ in CTLcom that describes property, the problem may be defined as a problem of establishing whether or not $M F=\varphi$, that is, $\forall \mathrm{s} \in I:(\mathrm{M}, \mathrm{s}) F=\varphi$.

A model that checks the CTLcom logic can be implemented in two methods:

- Direct method by either creating suitable model checker from scratch or expanding existent model checker using new algorithms for the required modalities like in $[6,36,47]$.

- Formal reduction into existent model checker like in [12, 35, 48].

This study follows the formal reduction method by transforming the model checking CTLcom problem into a problem of a model checking existent logic, which is called Generalized $C T L^{*}$, or simply GCTL* [13].

\subsection{Reduction Procedure}

This section shows how the CTLcom logic that is proposed by this paper can be a model checked by formally transforming the problem into a problem of a model that checks an existent logic, namely, GCTL*[13]. Consequently, we directly benefit from the

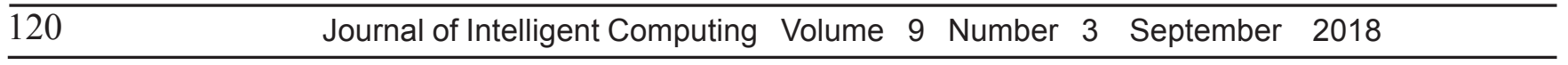


CWB-NC model checker1 of GCTL*. The section starts with a brief review of the GCTL* that expands the CTL* by allowing the formulae to restrain not only states, but also the actions. After that, we present the reduction ( also called transformation) procedure. The following BNF grammar defines the syntax of GCTL* as proposed in [13]:

$S::=p|\neg S| S \vee S \mid E P$

$P::=\theta|\neg P| S|P \vee P| X P \mid P \mathrm{U} P$

where $p$ is atomic proposition from $\phi p$ and $\theta$ is atomic action proposition from the set $\phi a$. Two types of formulae are distinguished: (i) state formulae $\mathrm{S}$ that hold on specific state; and (ii) path formulae $P$ that denote the temporal properties of the paths. State formulae are the legal GCTL* formulae. The GCTL* model is defined as follows.

Definition 5 (Model of GCTL*). A model $M G=(S G, A c t, l S, l A c t, \rightarrow, I G)$ is tuple where $S G$ is non-empty group of states; Act is group of actions; $1 \mathrm{~S}: S G \rightarrow 2 \phi p$ is state-labeling function; 1 Act : Act $\rightarrow 2 \phi a$ is action-labeling function; $\rightarrow \subseteq S G \times A c t \times S G$ is labeled transition relationship; and $I G \subseteq S G$ is group of initial states.

By intuition, $S G$ encompasses the reachable states of the system and Act expresses the atomic actions which the system may execute. In this sense, the labeling functions $\mathrm{IS}$ and $l A c t$ point to the atomic propositions that hold in a given state and action, respectively. The semantics of the GCTL* follow the standard convention in the temporal logic like CTL* [13]. A particular state fulfills $A \varphi$ (resp. $E \varphi$ ) if each path (resp. some paths) beginning from this state satisfies $\varphi$. Meantime, a certain path fulfills a state formula if the initial state in it does, and it fulfills $\theta$ if the label of the first transition on this path too fulfills $\theta$. The time operators $X$ and $U$ are as usual.

Our procedure of reducing the problem from a problem of a model that checks the CTLcom to the problem of a model that checks the $G C T L^{*}$ is defined as follows:

given a CTLcom model

$j \mid(i, j) \in A g 2\}, V)$ and

$M=\left(S, I, R t,\left\{{ }^{*} i\right.\right.$

a CTLcom formula $\varphi$, there is a need for defining a $G C T L^{*}$ model $M G=F(M)$ and a GCTL* formula $F(\varphi)$ using reduction function $F$ such that $M|=\varphi \operatorname{iff} F(M)|=F(\varphi)$. The model $F(M)$ is defined as GCTL* model $M G=(S G, A c t, l S, l A c t, \rightarrow, I G)$ as follows:

$$
\begin{aligned}
& S G=S \\
& I G=I ; \\
& l S=V ;
\end{aligned}
$$

For defining the set Act of actions, let us first define the set $\phi a$ of atomic action propositions from three types of actions: one for the transition relationship which is already present in the model, one for the relationship of social accessibility $\sim i \rightarrow j$ so as to capture the semantics of the commitment, and another for the symmetric closure of the social accessibility relationship $\sim i \rightarrow j$ in order to capture the semantics of fulfillment.

$\phi a=\{E, \alpha 1 \quad 1, \alpha, \ldots, \alpha\} \cup\{\gamma, \gamma, \ldots, \gamma\}$, then

Act $=\{\alpha 0, \alpha 11, \alpha 12, \ldots, \alpha n n\} \cup\{\gamma 11, \gamma 12, \ldots, \gamma n n\}$

where $\alpha_{o}$ and $\alpha_{i j}$ are actions that label the transitions defined respectively from the transition relation $R t$ and the social accessibility relation $\sim i \rightarrow j$, and $\gamma$ is action that labels the symmetric transition added when there is an added transition labeled with $\alpha_{i j}$ and needed to define the transformation of the formula $\mathrm{Fu}(\mathrm{Ci} \rightarrow \varphi)$;

The function $l A c t$ is then defined as follows:

${ }^{1} \mathrm{http}: / /$ www.cs.sunysb.edu/cwb/

Journal of Intelligent Computing Volume 9 Number 3 September 2018 
1. $\alpha_{0} \in$ Act, then $\operatorname{lAct}\left(\alpha_{0}\right)=\{E\}$,

2. $\operatorname{lAct}(\alpha i j)=\{\alpha i \rightarrow j\}$ for $1 \leq i \leq n$ and $1 \leq j \leq n$,

3. $\operatorname{lAct}(\gamma i j)=\{\gamma i \rightarrow j\}$ for $1 \leq i \leq n$ and $1 \leq j \leq n$;

- The labeled transition relationship $\rightarrow$ joins the temporal labeled transition $R t$, the accessibility relationships $\sim i \rightarrow j$, and the symmetric closure of the social accessibility relationship $\sim i \rightarrow j$ under the following situations: for states $s$ and $s 1 \in S$,

1. $(s, \alpha 0, s 1) \in \rightarrow$ if $(s, s 1) \in R t$,

2. $(s, \alpha \mathrm{ij}, s 1) \in \rightarrow$ if $s \sim I \rightarrow j s 1$,

3. $(s, \gamma i j, s 1) \in \rightarrow$ if $(s 1, \alpha i j, s) \in \rightarrow$.

Now, let us define $F(\varphi)$ as a $G C T L^{*}$ formula by induction on the form of the CTLcom formula $\varphi$.

$F(p)=p$, if $p$ is atomic proposition;

- $F(\neg \varphi)=\neg F(\varphi)$;

- $F(\mathrm{~V} \psi)=F(\varphi)(\mathrm{V} F(\psi)$;

- $F(E X \varphi)=E X F(\varphi)$;

- $F(E(\varphi \mathrm{U} \psi))=E(F(\varphi) U F(\psi))$;

- $F(E G \varphi)=E G F(\varphi)$;

- $F(C i \rightarrow \mathrm{j} \varphi)=\mathrm{A}(\alpha i \rightarrow j \Lambda X F(\varphi))$;

- $F(F u(C i \rightarrow j \varphi))=E(\gamma i \rightarrow \mathrm{j} \Lambda X F(C i \rightarrow j \varphi))$.

Figure 2 depicts an example illustrating the transformation function $F$.

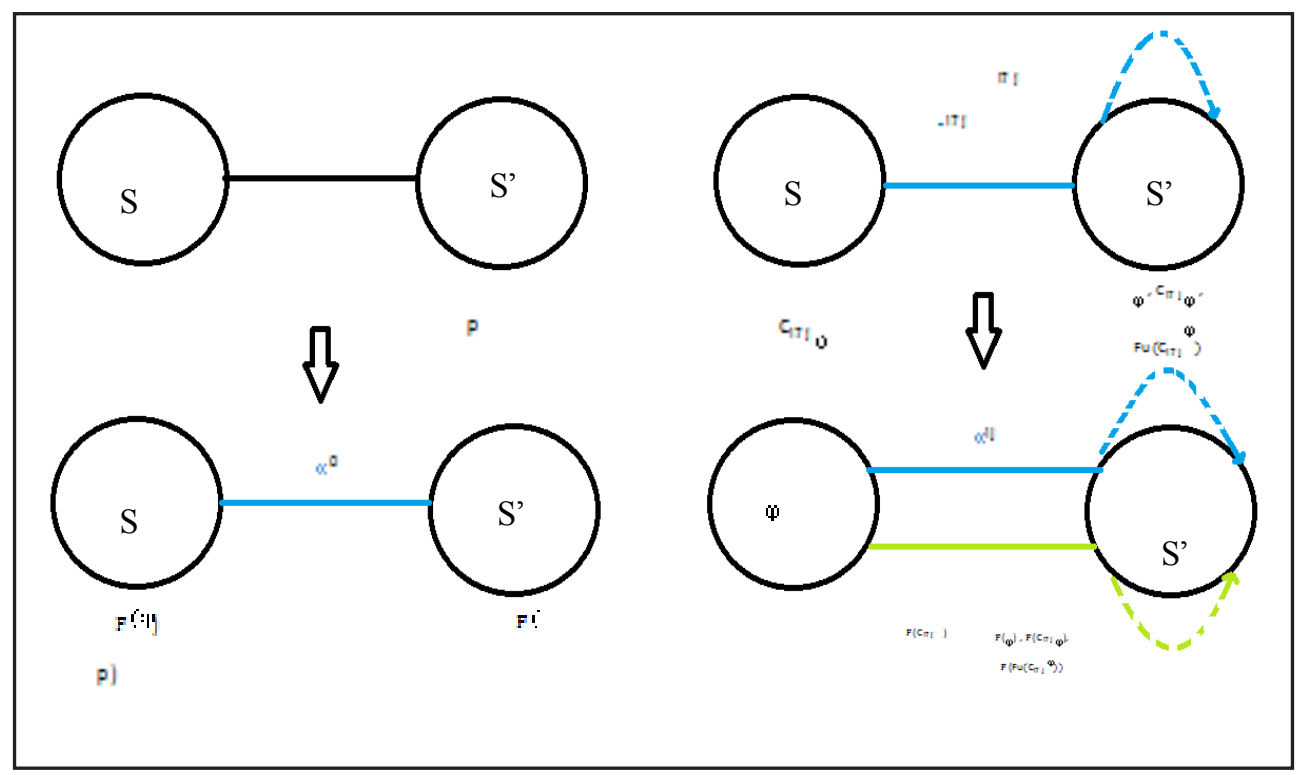

Figure 2. Example of the reduction function $F$

Theorem 1 (Soundness of $F$ ). Let $M$ and $\varphi$ be a CTLcom model and formula, respectively, and let $F(M)$ and $F(\varphi)$ be the 
associated model and formula in the $G C T L^{*}$. We have $M \mid=\varphi$ iff $F(M) \mid=F(\varphi)$.

Proof. We prove this theorem by induction on structure of the formula $\varphi$. All cases are straightforward, except the following two: ij

$\varphi=C i \rightarrow j \psi$. We have $(M, s) \mid=C i \rightarrow j \psi$ iff $(M, s 1) \mid=\psi$ for every $s 1 \in S$ such that $s \sim i \rightarrow j s 1$. Consequently, $(M, s) \mid=C i \rightarrow j \psi$ iff $(F$ $(\mathrm{M}), \mathrm{s} 1) \mid=\mathrm{F}(\psi)$ for every $s 1 \in S G$ such that $(s, \alpha, s 1) \in \rightarrow$. By the semantics of $A$ and $X$, we obtain $(F(M), s) \mid=\mathrm{A}(\alpha i \rightarrow j \wedge X F(\psi)$.

$\varphi=F u(C i \rightarrow j \psi)$. We have $(M, s) \mid=F u(C i \rightarrow j \psi)$ iff $(M, s 1) \mid=C i \rightarrow j \psi$ for state $s 1 \in S$ such that $s 1 \sim i \rightarrow j s$. Consequently, $(M, s)$ $\mid=F u(C i \rightarrow j \psi)$ iff $(F(M), s 1) \mid=F(C i \rightarrow j \psi)$ for $s 1 \in S G$ such that $(s, \gamma i j, s 1) \in \rightarrow$. Using the semantics of $E$ and $X$, we get:

$(F(M, s)) \mid=E(\gamma i \rightarrow j \Lambda X F(C i \rightarrow j \varphi))$

Therefore, the theorem.

\section{Case Study}

One of the main objectives of this part of this study was to test effectiveness of our proposed reduction technique. We have applied the reduction technique introduced in Section 5.1 atop of the GCTL* [13]. Additionally, the case study on which we could apply this technique was the NB protocol [44], which was formerly used in $[22,26,52]$ to illustrate how the commitments can specify protocols in the business settings.

\subsection{Modeling Net Bill Protocol}

The NetBill (NB) payment protocol is adopted as an e-commerce protocol for buying and selling encrypted software through the internet [44] as illustrated in Figure 3. In this protocol, two agents are interacting to reach to an agreement for buying and selling goods at low prices. The NB starts by authenticating one seller (merchant) and one buyer or more (customers). After that, the buyer asks for special prices for certain goods from the seller. The seller prepares an offer for the requested goods and sends it to the buyer. In this case, the buyer has two options: (i) either to accept the offer (i.e., to commit to pay); or (ii) to reject the offer. If the buyer accepts the offer, then the seller delivers the digital information (requested goods) encrypted prohibiting the key (i.e., he commits to deliver the due goods). Then, the buyer organizes electronic payment order (EPO) that includes description of the received goods (i.e., abides by her/his commitment). The EPO will be verified by the NB server. Furthermore, the NB server credits

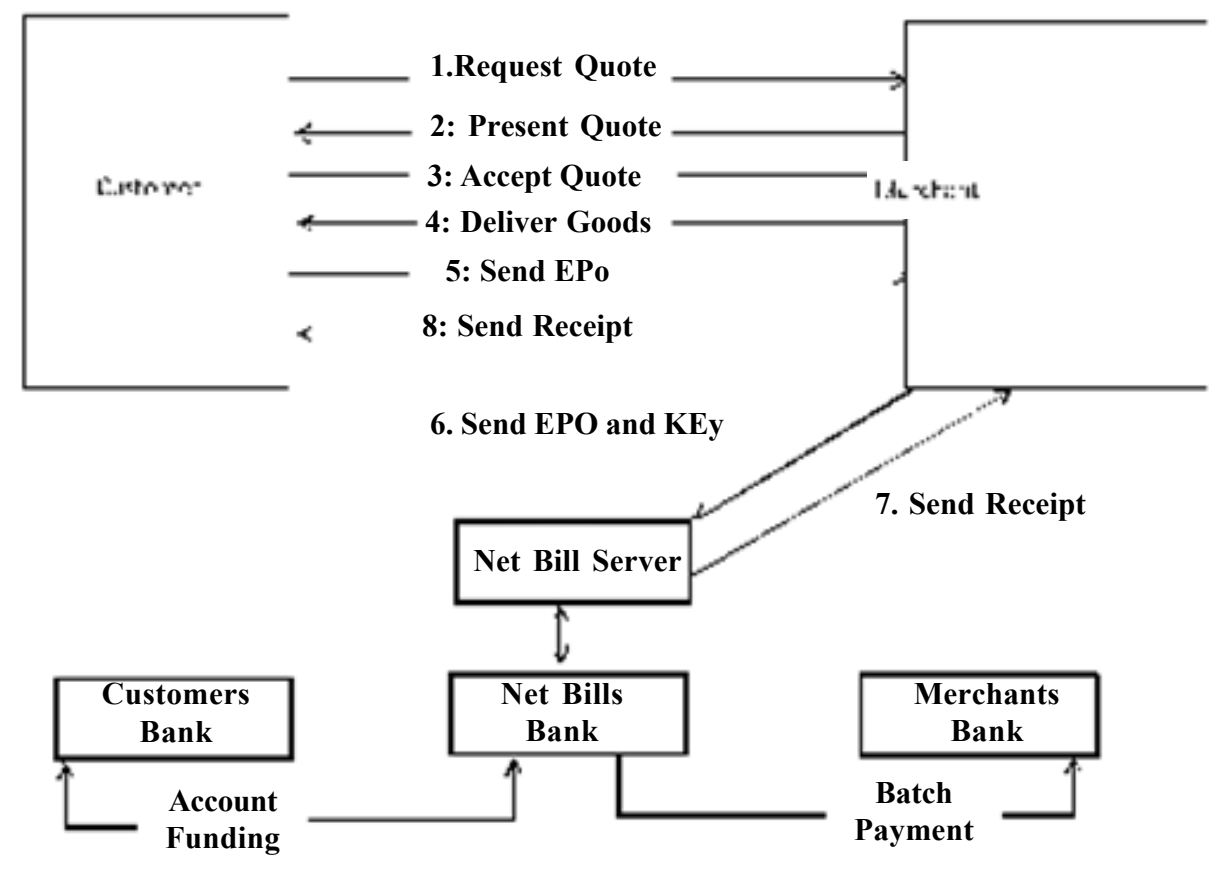

Figure 3. Example of the reduction function $F$ 
the sellers' account and issues a receipt including a key for decrypting the goods. Finally, the seller sends the receipts to the buyer (i.e., adheres to her/his commitments).

\subsection{Implementing the NetBill Protocol}

We processed the NB protocol using our reduction technique on top of model checker CWB-NC. This tool is based on the Alternating Bu" chi Tableau Automata (ABTA), which are a variant of the alternating tree automata like the non-deterministic and deterministic Bu"chi automata. The tool and a detailed user manual can be downloaded from: http://www.cs.sunysb.edu/ ${ }^{2}$ cwb/ DOWNLOADS/CWB/current/user.ps

In fact, the CWB-NC supports a variety of temporal logics (e.g., CTL, CTL*, and GCTL*). It also supports various languages of process algebra for specifying the behavior of the system like Milner's Calculus of Communicating Systems (CCS), a version of the CCS with prioritized actions (PCCS), and a version of the CCS with timed actions (TCCS). The attractive feature of the CWB$\mathrm{NC}$ is that it adopts an on-the-fly method in order for the algorithm to only search the segment of the state space that needs to be explored to prove or disprove a specific formula, that is, the space of the state is never established a priori. Recently, the CWB-NC has been employed in models checking large-scale, multi-agent interaction protocols known as the commitment protocols $[8,25]$.

In this paper, we used the CCS language to encode the specification of the $N B$ protocol presented in section 6.1 and formalized by the model $M=(S, I, R t,\{\sim i j \mid(i, j) \in A g 2\}, V)$.

Syntax of the CCS language is obtained from the following BNF grammar [13]:

$P::=\operatorname{nil}|\alpha . P|(P+P)|(P \mid P)| \operatorname{proc} C=P$

where $P$ denotes the CCS process and the process nil means no action at all.

If $P$ is process and $\alpha$ is an action prefixing, then $\alpha$. $P$ is process. Meanwhile, if $P 1$ and $P 2$ are processes, then so is $P 1+P 2$ using the choice operator "+", and if $P 1$ and $P 2$ are processes, then so is $P 1 \mid P 2$ using the parallel composition operator "". The keyword proc is employed to give the name $C$ to the process $P$.

In our own approach, the two agents of the NB protocol; customer (Cus) and merchant (M er), are encoded in the language CCS by defining every agent as a group of processes that recursively represent the local states of the agent. The commitment and fulfillment states of every agent are technically defined as variables in the proc statement. The actions of every agent are represented explicitly by using atomic action propositions in the proc statement so as to capture the labeled transitions in the states.

For instance, the customer Cus agent can be defined as follows:

$\operatorname{proc} \mathrm{Cus} 0=$ 'priceRequest.Cus 1

proc Cus $1=$ Offer.Cus 2

proc Cus2 $=\left({ }^{\prime}\right.$ Accept.Cus3 +'Reject.Cus4 $)$

which implies that the Cus agent makes a message of price request at the Cus0 state and develops into the Cus1 state. The M er agent replies to this customer by sending an offer message, which transforms the Cus agent into the Cus 2 state in which the Cus agent is willing to produce an acceptance message and enter into the state Cus3 state, or reject the message and enter into the state Cus4 state.

\subsection{Verification Results}

Our verification step was performed on a Toshiba Prote'ge' computer with $2.00 \mathrm{GHz}$ Intel Core Duo T6400 processor and 3GB memory under 64-bit Windows Vista Operating System. We report the results of 10 experiments in Table 1. We started our experiments with only two agents; the merchant (Mer) and customer (Cus), who interact in MAS to reason about their commitments and fulfillments. In the second experiment, we added one more customer. In the rest of our experiments, each time we added new agent up to 10 agents. In Table 1, the number of the reachable states, the number of transitions, and the memory usage are listed for the various numbers of agents examined. We noticed that the state space (expressed as the reachable states and transitions) increases exponentially with the number of agents. However, memory use increases polynomially with the number of agents, which shows effectiveness of our model checking method when the system scales up. The reason behind this increase in

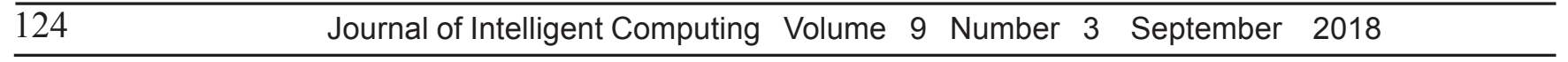


use of the memory when more agents are added is that the number of the reachable states and the mode size are increased.

Another fundamental objective of this paper was to use CTLcom to verify the properties of the protocols that involve interactions between the agents in the MASs by using the commitments and their fulfillments. Usually, these properties express certain

\begin{tabular}{|c|l|l|l|l|}
\hline \multicolumn{2}{|c|}{ Agent } & States & Transitions & Memory(MB) \\
\hline 2 & 1 (Mer.) +1 (Cust) & 24 & 72 & 4.778 \\
\hline 3 & 1 (Mer.) +2 (Cust) & 108 & 432 & 4.836 \\
\hline 4 & 1 (Mer.) $+3($ Cust) & 456 & 2280 & 4.945 \\
\hline 5 & 1 (Mer.) $+4($ Cust) & 1,184 & 11,304 & 5,157 \\
\hline 6 & 1 (Mer.) $+5($ Cust) & 7,696 & 53,872 & 5.727 \\
\hline 7 & 1 (Mer.) $+6($ Cust) & $3.13 \mathrm{E}+4$ & $250 \mathrm{E}+5$ & 6.400 \\
\hline 8 & 1 (Mer.) $+7($ Cust) & $1.27 \mathrm{E}+5$ & $1.14 \mathrm{E}+6$ & 6.892 \\
\hline 9 & 1 (Mer.) +8 (Cust) & $5.11 \mathrm{E}+5$ & $5.11 \mathrm{E}+6$ & 8.030 \\
\hline 10 & 1 (Mer.) $+9($ Cust) & $2.06 \mathrm{E}+6$ & $2.26 \mathrm{E}+7$ & 8.961 \\
\hline
\end{tabular}

Table 1. Verification results of the NetBill protocol using GCTL*

protocol requirements that need to be met. In effect, several properties are proposed in the literature $[15,17,7,27]$. In this section, we check the Safety, Liveness, Reachability, and Commitment Fulfillment properties in the NB protocol.

\section{Safety}

The safety property means ensuring that 'something bad will never take place'. In general, this property can be expressed in the CTL using the formula $A G \neg \varphi$, where $\varphi$ denotes a bad situation. The bad situations encircle, as an example, the case when the customer (Cus) sends the payment for the merchant (Mer) (i.e., she/he fulfills meets her/his commitment), but the merchant does not send receipt to her/him. This bad situation can be avoided using CTLcom as follows:

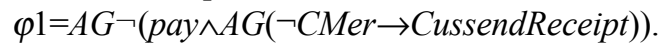

\section{Liveness}

This property means implies that 'eventually something good will take place'. For instance, in all the paths, globally, when customer asks for a price offer, then in all the paths in the future the merchant will deliver the due goods. This property is expressed in the CTLcom as follows:

$\varphi 2=A G($ reqOffer $\rightarrow A F(C M e r \rightarrow$ CusdeliverGoods $))$

\section{Reachability}

This property indicates that some particular situation can be reached. It takes the form $E F \varphi$ where $\varphi$ is the situation due to be reached. For example, the merchant will eventually commit to the customer on delivering the required goods, which should be reached from the initial state. This property may be expressed as follows:

$\varphi 3=E F(C M$ er $\rightarrow$ CusdeliverGoods $)$

\section{Fulfillment of Commitment}

When verifying the behaviors of the agents in terms of fulfillment of their commitments, it is critical to verify some of the conditions under which fulfillment may take place. For instance, when a customer sends payment to the merchant, the commitment is fulfilled successfully. This property can be expressed as follows:

$\varphi 4=E F(F U(C C u s \rightarrow$ Mer Pay $))$

Table 2 lists the outcomes of model check of the aforementioned desirable properties in the case of the NB protocol. 


\begin{tabular}{|c|c|c|}
\hline Formulae & Results & Time for MC (sec) \\
\hline$? 1$ & True & $<0.01$ \\
$? 1$ & True & $<0.01$ \\
$? 1$ & True & $<0.01$ \\
$? 1$ & True & $<0.01$ \\
\hline
\end{tabular}

Table 2. Results of model checking some protocol properties

We notice that the four formulae hold in the model, hence suggesting that that our approach is successful in expressing the properties of the protocol using CTLcom and that our reduction-based model checking technique works effectively. The time that elapsed in verifying each of the formulae is close to zero and is almost the same for each tested formulae. This indicates efficiency of the underlying algorithms and the optimization methods that were employed in the CWB-NC model checker.

\section{Conclusions and Suggestions for Future Work}

This paper introduced new, automatic method for trimming down the problem of a model that checks the CTLcom, which is an extension of the CTL with modalities for the commitments and their fulfillment. This proposed method constructs a group of reduction rules so as to formally reduce the problem of a model that checks the CTLcom to that of a model that checks the GCTL*. Effectiveness of this technique was assessed by testing it on a real case study drawn from the e-business field, that is, the NB protocol, and running the reduction tool atop of the CWB-NC model checker. The results proved soundness of this reduction technique. We could successfully check some of the desirable properties of the protocol expressed in the CTLcom. Moreover, we showed that the system is scalable since we could check up to $2.06 \mathrm{E}+6$ states and $2.26 \mathrm{E}+7$ transitions. These promising outcomes confirm effectiveness of the CTLcom in capturing the interactions between the agents by utilizing the social commitment concepts, even in large systems.

In the future, we plan to develop model checking algorithms that are dedicated for the CTLcom logic and apply them atop of the MCMAS+ symbolic model checker. By doing this, we will become able to compare between the two techniques (i.e., the reduction and model checking algorithms) by using the obtained verification results. Additionally, we plan to take into consideration other actions like withdrawal, violation, delegation, and assignment. We also plan to examine the interactions between the agents in the MASs by using probabilistic communicative commitments.

\section{Acknowledgments}

Our thanks to Al al-Bayt University which has contributed towards the development of this research through a sabbatical leave.

\section{References}

[1] Alberti, M., Gavanelli, M., Lamma, E., Mello, P., Torroni. P. 92004). Specification and verification of agent interaction using social integrity constraints. Electr. Notes Theor. Comput. Sci., 85 (2) 94-116, .

[2] Anger, F. D., Clarke, E. M. (1993). New and used temporal models: An issue of time. Applied Intelligence, 3(1) 5-15.

[3] Baier, C., Katoen, J.-P. (2008). Principles of Model Checking (Representation and Mind Series). The MIT Press.

[4] Baldoni, M., Baroglio, C., Marengo. E. (2010). Behavior-oriented commitment-based protocols. In: ECAI, p. 137-142.

[5] Be'he', F., Galland, S., Gaud, N., Nicolle, C., Koukam, A. (2014). An ontology-based metamodel for multiagent- based simulations. Simulation Modelling Practice and Theory, 40. 64-85.

[6] Bentahar, J., El-Menshawy, M., Qu, H., Dssouli, R. (2012). Communicative commitments: Model checking and complexity analysis. Knowledge-Based Systems, 35. 21-34.

[7] Bentahar, J., Meyer, J.-J., Wan, W. (2009). Model checking communicative agent-based systems. Know.-Based Syst., 22(3)

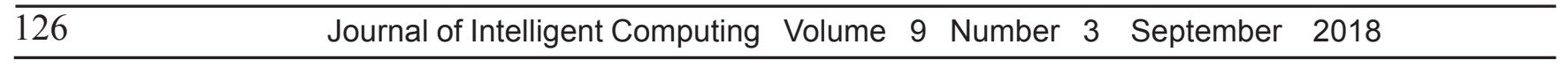


142-159, April.

[8] Bentahar, J., Meyer, J.-J., Wan, W. (2010). Model checking agent communication. In: Specification and Verification of Multiagent Systems, p. 67-102. Springer.

[9] Bentahar, J., Moulin, B., Chaib-draa. B. (2003). Towards a formal framework for conversational agents. In: Proceedings of the Agent Communication Languages and Conversation Policies AAMAS 2003 Workshop, 2003. July $14^{\text {th }} 2003$.

[10] Bentahar, J., Moulin, B., Meyer, J.-J. C., Chaib-draa. B. (2004). A logical model for commitment and argu- ment network for agent communication. In: Proceedings of the Third International Joint Conference on Autonomous Agents and Multiagent Systems, AAMAS '04, p. 792-799, 2004.

[11] Bentahar, J., Moulin, B., Meyer, J.-J. C., Lespe'rance, Y. (2007). A new logical semantics for agent communication. In: Proceedings of the $7^{\text {th }}$ International Conference on Computational Logic in Multi-agent Systems, p. 151-170, Berlin, Heidelberg. Springer-Verlag.

[12] Bentahar, J., Yahyaoui, H., Kova, M., Maamar. Z. (2013). Symbolic model checking composite web services using operational and control behaviors. Expert Syst. Appl., 40 (2) 508-522.

[13] Bhat, G., Cleaveland, R., Groce, A. (2001). Efficient model checking via Büchi tableau automata. In: G. Berry, H. Comon, and A. Finkel, editors, CAV, Lecture Notes in Computer Science, p. 38-52. Springer.

[14] Castelfranchi. C. (1995). Commitments: From individual intentions to groups and organizations. In: V. R. Lesser and L. Gasser, editors, ICMAS, p. 41-48. The MIT Press.

[15] Cheng, Z. (2006). Verifying commitment-based business protocols and their compositions: model checking using promela and spin. North Carolina State University, Ph.D. thesis.

[16] Clarke, E. M., Grumberg, O., Peled, D. A. (1999). Model checking. The MIT Press, Cambridge.

[17] Desai, N., Cheng, Z., Chopra, A. K., Singh, M. P. (2007). Toward verification of commitment protocols and their compositions. In: Proceedings of the International Conference on Autonomous Agents and Multiagent Systems (AAMAS), p 144-146.

[18] Desai, N., Chopra, A. K., Singh. M. P. (2009). Amoeba: A methodology for modeling and evolving cross- organizational business processes. ACM Trans. Softw. Eng. Methodol., 19 (2).

[19] Dignum, F., Greaves, M. (2000). editors. Issues in Agent Communication, volume 1916 of Lecture Notes in Computer Science. Springer.

[20] Dignum, F., Meyer, J.-J. C., Wieringa, R., Kuiper. R. (1996). A modal approach to intentions, commitments and obligations: Intention plus commitment yields obligation. In DEON, p. 80-97, 1996.

[21] Dourlens, S., Ramdane-Cherif, A., Monacelli. E. (2013). Multi levels semantic architecture for multimodal interaction. Applied Intelligence, 38 (4) 586-599.

[22] El-Menshawy, M., Bentahar, J., Dssouli, R. (2010). Symbolic model checking commitment protocols using reduction. In: DALT, p. 185-203.

[23] El-Menshawy, M., Bentahar, J., Dssouli. R. (2010). Verifiable semantic model for agent interactions using social commitments. In: LADS, p 128-152.

[24] El-Menshawy, M., Bentahar, J., Dssouli. R. (2010). Verifiable semantic model for agent interactions using social commitments. In: LADS, p 128-152.

[25] El-Menshawy, M., Bentahar, J., Kholy, W. E., Dssouli, R. (2013). Reducing model checking commitments for agent communication to model checking ARCTL and GCTL*. Autonomous Agents and Multi-Agent Systems, 27 (3) 375-418.

[26] El-Menshawy, M., Bentahar, J., Kholy, W. E., Dssouli. R. (2013). Verifying conformance of multi-agent commitment-based protocols. Expert Syst. Appl., 40 (1) 122-138.

[27] El-Menshawy, M., Bentahar, J., Qu, H., Dssouli. R. (2011). On the verification of social commitments and time. In: Proceedings of the International Conference on Autonomous Agents and Multiagent Systems (AAMAS), p 483-490. 
[28] El-Menshawy, M., Bentahar, J., Qu, H., Dssouli, R. (2011). On the verification of social commitments and time. In AAMAS, $p 483-490$.

[29] Fagin, R., Halpern, J. Y., Moses, Y., Vardi, M. Y. (1995). Reasoning about Knowledge. The MIT Press, Cambridge.

[30] Fornara, N., and Colombetti, M. (2004). A commitment-based aproach to agent communication. Applied Artificial Intelligence, 18 (9-10): 853-866.

[31] Fornara, N., Vigano', F., Verdicchio, M., Colombetti, M. (2008). Artificial institutions: a model of institutional reality for open multiagent systems. Artif. Intell. Law, 16 (1):89-105.

[32] Gnay, A., Yolum, P. (2013). Constraint satisfaction as a tool for modeling and checking feasibility of multiagent commitments. Applied Intelligence, 39(3): 489-509, 2013.

[33] Günay, A., Yolum. P. (2013). Constraint satisfaction as a tool for modeling and checking feasibility of multiagent commitments. Applied Intelligence, 39 (3): 489-509, 2013.

[34] Konur, S., Fisher, M., Schewe, S. (2013). Combined model checking for temporal, probabilistic, and real-time logics. Theor. Comput. Sci., 503: 61-88, 2013.

[35] Lomuscio, A., Pecheur, C., Raimondi. F. (2007). Automatic verification of knowledge and time with nusmv. In Proceedings of the $20^{\text {th }}$ International Joint Conference on Artifical Intelligence, IJCAI'07, p1384-1389, San Francisco, CA, USA, 2007. Morgan Kaufmann Publishers Inc.

[36] Lomuscio, A., Penczek, W. (2012). Symbolic model checking for temporal-epistemic logic. In Logic Programs, Norms and Action, $p$ 172-195.

[37] Mallya, A. U., Singh. M. P. (2007). An algebra for commitment protocols. Autonomous Agents and Multi-Agent Systems, 14(2): 143-163.

[38] Searle, J. R. (1969). Speech acts: An essay in the philosophy of language. Cambridge, Cambridge University Press, 1969.

[39] Singh, M. P. (1991). Social and psychological commitments in multiagent systems. In: AAAI Fall Symposium on Knowledge and Actions at Social and Organizational Levels, $p$ 104-106, 1991.

[40] Singh. M. P. (1996). A conceptual analysis of commitments in multiagent systems. Technical report, Raleigh, NC, USA.

[41] Singh, M. P. (1998). Agent communication languages: Rethinking the principles. IEEE Computer, 31 (12), 40-47.

[42] Singh, M. P. (1999). An ontology for commitments in multiagent systems. Artif. Intell. Law, 7(1):97-113.

[43] Singh, M. P. (2000). A social semantics for agent communication languages. In Issues in Agent Communication, p 31-45, 2000.

[44] Sirbu, M. A. (1997). Credits and debits on the internet. IEEE Spectr., 34(2): 23-29, Feb.

[45] Venkatraman, M., Singh, M. P. (1999). Verifying compliance with commitment protocols. Autonomous Agents and MultiAgent Systems, 2 (3): 217-236.

[46] Verdicchio, M., Colombetti. M. (2003). A logical model of social commitment for agent communication. In: Proceedings of the second international joint conference on Autonomous agents and multiagent systems, AAMAS '03, p 528-535. ACM.

[47] Wan, W., Bentahar, J., Hamza, A. B. (2011). Model checking epistemic and probabilistic properties of multi-agent systems. In IEA/AIE (2), p 68-78.

[48] Wan, W., Bentahar, J., Hamza. A. B. (2013). Model checking epistemic-probabilistic logic using probabilistic interpreted systems. Knowl.-Based Syst., 50: 279-295.

[49] Wooldridge, M. (2002). Introduction to multiagent systems. Wiley.

[50] Wooldridge, M., Jennings, N. R. (1995). Intelligent agents: Theory and practice. Knowledge Engineering Review, 10 (2), 115152 .

[51] Yolum, P. (2007). Design time analysis of multiagent protocols. Data Knowl. Eng., 63(1):137-154, October.

[52] Yolum, P., Singh, M. P. (2004). Reasoning about commitments in the event calculus: An approach for specifying and executing protocols. Ann. Math. Artif. Intell., 42 (1-3): 227-253.

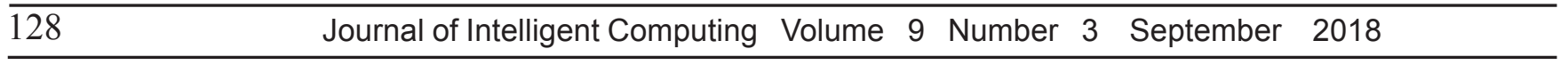

